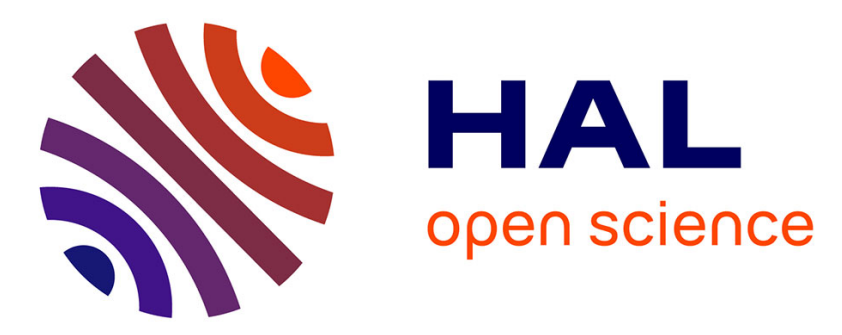

\title{
Étude du frottement intérieur des borates et phosphoaluminates vitreux par la méthode d'écho d'impulsion (pulse-echo)
}

\author{
J. Phalippou, Alexandre Boyer, E. Groubert, J. Zarzycki
}

\section{To cite this version:}

J. Phalippou, Alexandre Boyer, E. Groubert, J. Zarzycki. Étude du frottement intérieur des borates et phosphoaluminates vitreux par la méthode d'écho d'impulsion (pulse-echo). Revue de Physique Appliquée, 1975, 10 (6), pp.437-442. 10.1051/rphysap:01975001006043700 . jpa-00243943

HAL Id: jpa-00243943

https://hal.science/jpa-00243943

Submitted on 1 Jan 1975

HAL is a multi-disciplinary open access archive for the deposit and dissemination of scientific research documents, whether they are published or not. The documents may come from teaching and research institutions in France or abroad, or from public or private research centers.
L'archive ouverte pluridisciplinaire HAL, est destinée au dépôt et à la diffusion de documents scientifiques de niveau recherche, publiés ou non, émanant des établissements d'enseignement et de recherche français ou étrangers, des laboratoires publics ou privés. 


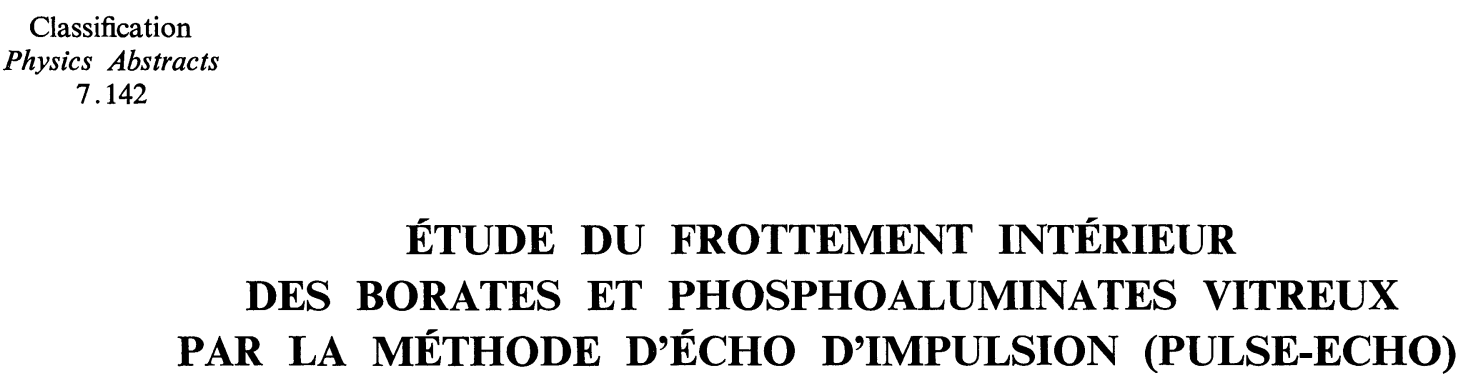

\author{
J. PHALIPPOU, A. BOYER $(*)$, E. GROUBERT $\left({ }^{*}\right)$ et J. ZARZYCKI
}

Laboratoire de Science des Matériaux et Laboratoire des Verres du C. N. R. S. Université des Sciences et Techniques du Languedoc, 34060 Montpellier Cedex, France

(Reçu le 26 mai 1975, accepté le 23 juin 1975)

\begin{abstract}
Résumé. - Les mesures d'atténuation et de vitesse des ultrasons, en fonction de la température ont été réalisées par la méthode de l'écho d'impulsion ("pulse-echo ») dans deux verres de borate et d'aluminophosphate de sodium. L'analyse du pic attribué à la diffusion sous contrainte des ions sodium a été faite par décomposition en partie réelle et imaginaire des modules d'élasticité pour des ondes longitudinales et des ondes transverses. En supposant une distribution log-normale des temps, de relaxation, on examine la variation de $\beta$, paramètre de cette distribution, en fonction du mode de propagation et de la fréquence de l'onde ultrasonore.
\end{abstract}

\begin{abstract}
The attenuation and speed of ultrasound were measured as a function of temperature in two sodium borate and phosphoaluminate glasses by means of a pulse-echo method. The peak corresponding to the stress diffusion of sodium ions was analysed by separating the real and imaginary parts of the moduli of elasticity for both longitudinal and transverse waves. Assuming a lognormal distribution of relaxation times, the variation of the parameter $\beta$ of this distribution was studied as a function of the mode of propagation and frequency of the ultrasonic waves.
\end{abstract}

1. Introduction. - Les courbes de frottement interne des verres alcalins en fonction de la température présentent un pic de relaxation. Ce pic, attribué à la diffusion sous contrainte des ions alcalins a été étudié au moyen de trois méthodes différentes: Pendule de torsion $(1 \mathrm{~Hz})$; Méthode du barreau résonnant (1 $\mathrm{kHz}$ ) et Méthode de l'écho d'impulsion (pulseecho, $10 \mathrm{MHz}$ ).

Quelle que soit la méthode employée, le pic de relaxation est de hauteur trop faible et de largeur trop importante pour être assimilé à une courbe de Debye. Plusieurs auteurs [1, 2] ont montré que la forme de courbe nécessiterait une distribution des temps de relaxation. Une distribution log-normale des temps de relaxation a été utilisée avec succès pour décrire le pic de relaxation sous contrainte des ions sodium $[3,4,5]$.

En utilisant une variable $z=\log \left(\tau / \tau_{\mathrm{m}}\right)$ où $\tau_{\mathrm{m}}$ est la valeur la plus probable du temps de relaxation, la fonction de distribution normalisée $\psi$ est définie par :

(*) Centre d'Etude d'Electronique des Solides, Laboratoire associé au C. N. R. S., n 21.

$$
\psi(Z)=\frac{1}{\beta \sqrt{\pi}} \exp \left(-(Z / \beta)^{2}\right)
$$

où $\beta$ est le paramètre de cette distribution.

Nowick et Berry [6] montrent que $\beta$ est lié à la largeur à mi-hauteur de la courbe, partie imaginaire du module en fonction de $1 / T$. Dans une publication précédente [5] nous avons montré que ce paramètre $\beta$ variait avec la température du pic, ou ce qui revient au même, avec la fréquence utilisée pour déterminer la courbe de frottement intérieur. La variation de $\beta$ définie à partir des résultats obtenus au moyen d'appareils différents peut être différemment exploitée et interprétée. En effet, puisque les trois contraintes appliquées au matériau font intervenir trois modules différents, il est possible que la variation de $\beta$ soit due au mode de déformation du matériau. Donc seules des mesures réalisées avec la même méthode, à la même fréquence et avec la même précision, mais faisant intervenir des modules différents, permettraient d'apporter une réponse.

Nous avons adopté la méthode de l'écho d'impulsion. Cette méthode est particulièrement adaptée à de 
telles études puisque suivant la coupe du quartz émetteur on peut créer des ondes longitudinales ou des ondes transverses.

La propagation des ondes longitudinales dans un milieu dont les dimensions latérales sont grandes visà-vis de la longueur d'onde permet de calculer le module de compression uniaxiale, $M^{*}$ :

$$
M^{*}=M^{\prime}+\mathrm{i} M^{\prime \prime}
$$

où $M^{\prime}$ et $M^{\prime \prime}$ sont respectivement les parties réelles et imaginaires du module complexe $M^{*}$. $M^{\prime}$ et $M^{\prime \prime}$ sont liés aux atténuations et aux vitesses par les relations :

$$
\begin{aligned}
& M^{\prime}=\rho v_{\mathrm{L}}^{2} \frac{1-r^{2}}{\left[1+r^{2}\right]^{2}} \\
& M^{\prime \prime}=2 \rho v_{\mathrm{L}}^{2} \frac{r}{\left[1+r^{2}\right]^{2}}
\end{aligned}
$$

où $v_{\mathrm{L}}$ est la vitesse de propagation des ondes longitudinales exprimée en $\mathrm{cm} / \mathrm{s}, \rho$ la masse volumique du matériau à la température considérée et $r$ est égal à $\alpha \lambda / 2 \pi$ où $\lambda$ est la longueur d'onde et $\alpha$ l'atténuation exprimée en néper $/ \mathrm{cm}$.

De même, une onde de cisaillement permet la mesure du module complexe :

$$
G^{*}=G^{\prime}+\mathrm{i} G^{\prime \prime} .
$$

Les relations sont identiques à celles déjà écrites, $\alpha_{\mathrm{T}}$ et $v_{\mathrm{T}}$ étant maintenant les atténuations et vitesses des ondes transverses. Le module de compression volumique $K^{*}$ est lié aux deux modules définis plus haut par la relation :

$$
K^{*}=M^{*}-\frac{4}{3} G^{*} .
$$

Dans ces conditions, un calcul précis de $K^{\prime \prime}$ ne peut se faire que si $G^{\prime \prime}$ est inférieur à $M^{\prime \prime}$; en effet, si $M^{\prime \prime}$ et $G^{\prime \prime}$ sont voisins, l'erreur commise sur $K^{\prime \prime}$ peut devenir importante [7].

2. Technique expérimentale. - Les techniques utilisées pour la mesure de l'atténuation et de la vitesse en écho d'impulsion ont été largement décrites [8, 9]. Toutefois, pour des températures comprises entre $400^{\circ} \mathrm{C}$ et $450^{\circ} \mathrm{C}$, le quartz n'est plus collé directement sur l'échantillon, mais par l'intermédiaire d'un barreau en silice (Suprasil) de $32 \mathrm{~cm}$ de long, $2,6 \mathrm{~cm}$ de diamètre et rainuré sur sa longueur pour éliminer les échos parasites. La mesure de l'atténuation des ultrasons doit alors être corrigée en tenant compte de l'atténuation du barreau en silice et des pertes acoustiques par diffraction qui restent constantes dans l'intervalle de température considéré. Nous avons pu, ainsi, compléter les courbes d'atténuations par des mesures relatives. Pour transmettre l'onde de déformation créée par le quartz, il est nécessaire de lier celui-ci à l'échantillon par un corps intermédiaire. Un certain nombre de liants ont été choisis suivant le domaine de température et le mode de propagation de l'onde (Tableau I).

\section{TABLEAU I}

Liaisons utilisées

$\begin{array}{ccccc}\text { Intervalle de } & -200 & 20 & 200 & 400 \\ \text { Température }\left({ }^{\circ} \mathrm{C}\right) & 25 & 200 & 400 & 450 \\ \text { Onde longitudi- } & \text { Nonaq Stopcock } & \text { D. C. } & \text { D. C. } & \mathrm{AgCl} \\ \text { nale } & \text { Grease } & 710 & 710 & \\ \text { Onde transverse } & \text { Convalex 10 } & \text { Celvaseal } & \mathrm{AgCl} & \mathrm{AgCl}\end{array}$

3. Echantillons. - Les deux verres suivants ont été étudiés (compositions données en fractions molaires).

$$
\begin{array}{ll}
\text { Verre A } & 0,66 \mathrm{~B}_{2} \mathrm{O}_{3}-0,33 \mathrm{Na}_{2} \mathrm{O} \\
\text { Verre B } & 0,48 \mathrm{P}_{2} \mathrm{O}_{5}-0,05 \mathrm{Al}_{2} \mathrm{O}_{3}-0,47 \mathrm{Na}_{2} \mathrm{O} .
\end{array}
$$

Le verre de diborate de sodium (A) est préparé dans un creuset en platine $10 \%$ rhodium à partir de $\mathrm{B}_{2} \mathrm{O}_{3}$ déshydraté et pur et de $\mathrm{Na}_{2} \mathrm{CO}_{3}$ (R. P.). La fusion est maintenue pendant trois heures à $1000^{\circ} \mathrm{C}$ pour l'affinage du verre. Le verre de phosphoaluminate de sodium (B) est préparé en deux étapes. L'alumine, sous forme d'hydroxyde pur est ajoutée au verre de base obtenu par fusion de $\mathrm{NaH}_{2} \mathrm{PO}_{4}-2 \mathrm{H}_{2} \mathrm{O}$ (R. P.) dans un creuset Pythagoras. Les verres sont coulés dans un moule en acier. La température de transition vitreuse est déterminée par A. T. D. et ainsi ces verres sont recuits à une température de $20^{\circ} \mathrm{C}$ au-dessous de leur $\mathrm{Tg}$ pendant 4 heures et refroidis ensuite lentement jusqu'à la température ambiante.

Les échantillons sont cylindriques et ont un diamètre de $15 \mathrm{~mm}$. L'épaisseur de l'échantillon varie de $5 \mathrm{~mm}$ pour les phosphates, à $7 \mathrm{~mm}$ pour les borates. Les faces des échantillons sont polies et parallèles avec une tolérance de 5 secondes d'arc.

La densité des échantillons a été mesurée par la méthode d'Archimède dans du benzène pour spectroscopie à $16^{\circ} \mathrm{C}$. La densité du benzène était mesurée ensuite par picnométrie à la même température. La référence utilisée était l'eau déionisée bouillie et froide.

Les résultats obtenus sont:

$$
\begin{array}{cl}
\text { Verre A } & \rho=2,389 \pm 0,003 \mathrm{~g} / \mathrm{cm}^{3} \\
\text { Verre B } & \rho=2,574 \pm 0,003 \mathrm{~g} / \mathrm{cm}^{3} .
\end{array}
$$

La mesure des coefficients de dilatation thermique des verres $\mathrm{A}$ et $\mathrm{B}$ a été réalisée sur un dilatomètre différentiel mécanique ADAMEL Type $T$ 50. La référence est une baguette de Pyros coulée 58 dont les coefficients de dilatation thermique à chaque température sont connus. Les échantillons sont des parallélépipèdes de $65 \mathrm{~mm}$ de long et de base carrée de $3 \mathrm{~mm}$ de côté. Les coefficients de dilatation moyens $a_{\mathrm{m}}$ mesurés dans la partie linéaire de la courbe sont les suivants : 
Intervalle de température $\quad a_{\mathrm{m}}$ degré $^{-1}$

Verre A $20-400{ }^{\circ} \mathrm{C}$

Verre B $20-280^{\circ} \mathrm{C}$

$16,3 \times 10^{-6}$

$19,7 \times 10^{-6}$

4. Résultats expérimentaux. - 4.1 AtTÉNUATION ET VITESSE DES ONDES ULTRASONORES. - Les courbes (Fig. 1a) relatives au verre A montrent la varia-

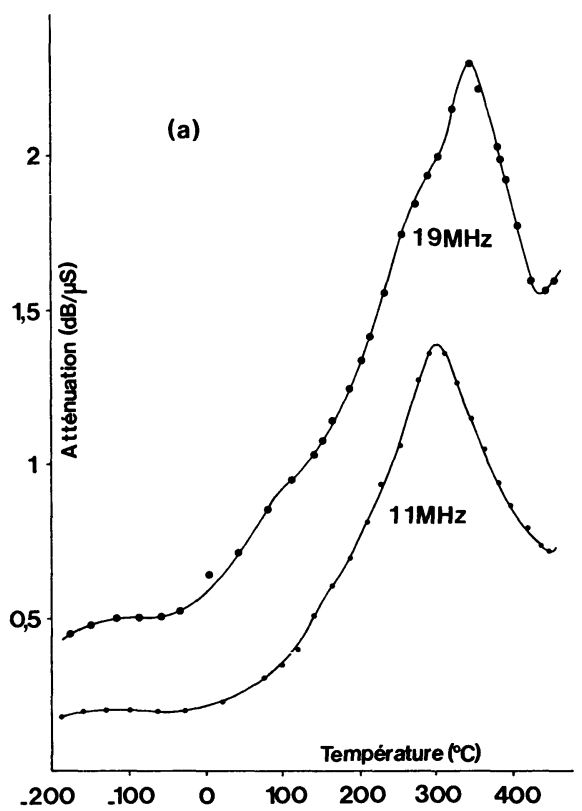

Fig. 1a. - Atténuation des ondes ultrasonores longitudinales en fonction de la température dans le verre $\mathrm{A}$ pour les fréquences de 11 et $19 \mathrm{MHz}$.

tion de l'atténuation des ondes longitudinales en fonction de la température pour les deux fréquences 11 et $19 \mathrm{MHz}$. La figure $1 b$ est relative à la mesure de l'atténuation des ondes transverses pour une fréquence de $11 \mathrm{MHz}$. Aucun effet ne se produit aux basses

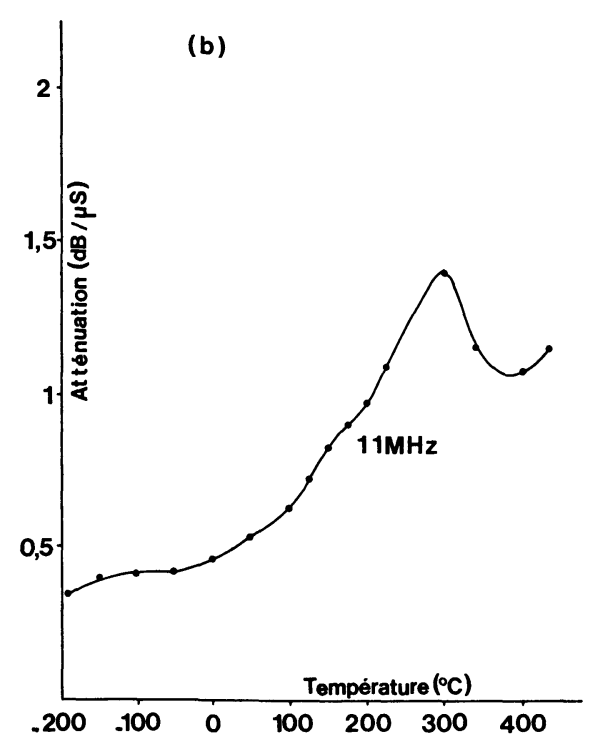

FIG. 1b. - Atténuation des ondes ultrasonores transverses en fonction de la température dans le verre A pour la fréquence de $11 \mathrm{MHz}$. températures, tandis que vers les hautes températures apparaît le pic de relaxation sous contrainte des ions sodium. Avec l'augmentation de la fréquence le pic se déplace vers des températures plus élevées.

La vitesse de propagation des ondes a été corrigée en fonction de la dilatation linéaire de l'échantillon (Fig. 2) pour la fréquence de $11 \mathrm{MHz}$. La vitesse décroît avec l'augmentation de la température; les vitesses longitudinales sont sensiblement égales à deux fois les vitesses transverses.

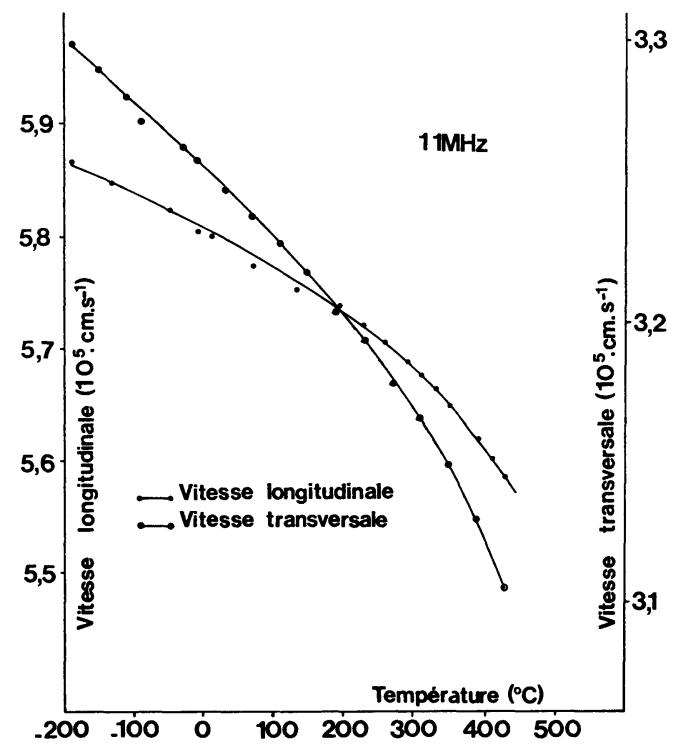

FIG. 2. - Variation des vitesses transverses et longitudinales en fonction de la température pour la fréquence $11 \mathrm{MHz}$.

Les valeurs des vitesses longitudinales obtenues pour le verre A confirment les travaux de Kurkjian et Krause [10] sur des composés similaires.

Pour le verre B, seule l'atténuation des ondes longitudinales a pu être mesurée pour les fréquences de 11 et $19 \mathrm{MHz}$ (Fig. 3). Le pic de relaxation est mieux résolu pour la fréquence la plus basse.

La vitesse des ondes longitudinales pour $11 \mathrm{MHz}$ montre un effet comparable à celui obtenu avec le verre $\mathrm{A}$.

4.2 ÉNERGIE D'ACTIVATION APPARENTE DES IONS sodium. - $\mathrm{Si}$ on suppose que le temps de relaxation suit une loi d'Arrhénius :

$$
\tau=\tau_{0} \exp \cdot \frac{E_{\mathrm{A}}}{R T}
$$

il est alors possible de calculer une énergie d'activation apparente pour ce mécanisme de diffusion sous contrainte des ions sodium dans les deux verres $\mathrm{A}$ et $\mathrm{B}$. Au sommet du pic $\omega \tau_{\mathrm{m}}=1$, de sorte que, si le maximum apparaît aux températures $T_{1}$ et $T_{2}$ pour les fréquences respectives $f_{1}$ et $f_{2}$

$$
E_{\mathrm{A}}=\frac{R \log f_{2} / f_{1}}{\frac{1}{T_{1}}-\frac{1}{T_{2}}}
$$




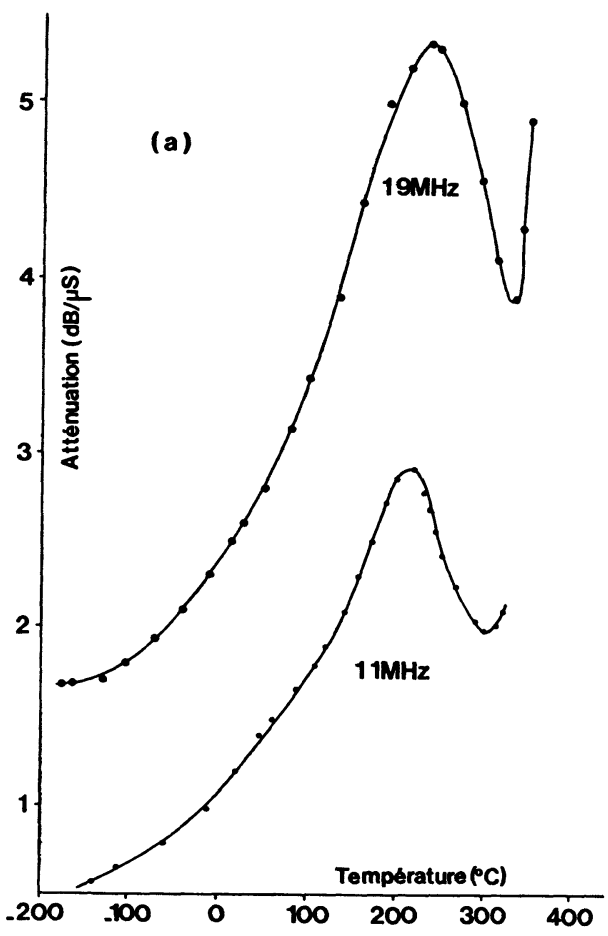

FIG. 3a. - Atténuation des ondes ultrasonores longitudinales dans le verre B pour les deux fréquences 11 et $19 \mathrm{MHz}$.

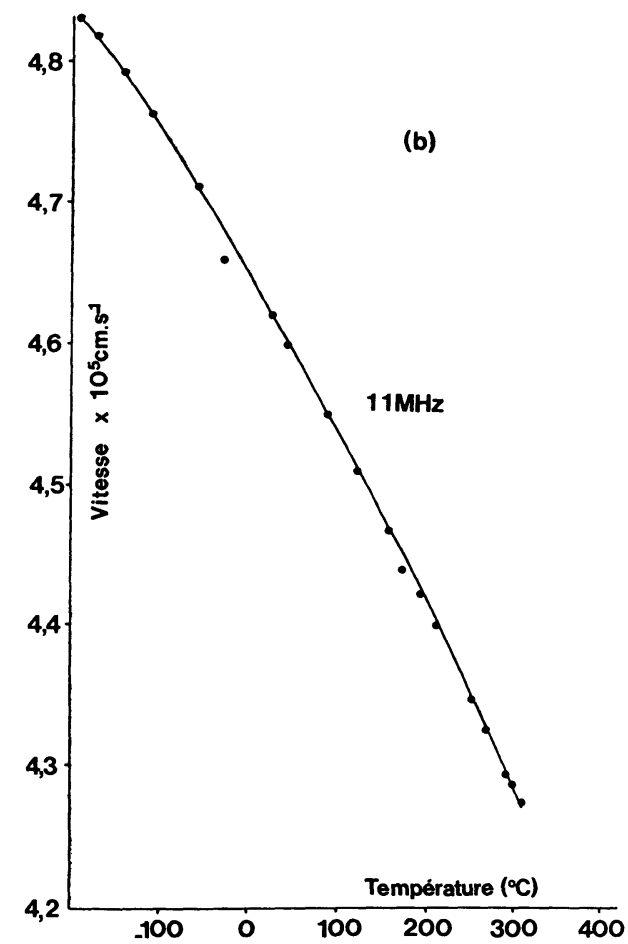

Fig. $3 b$. - Variation de la vitesse des ondes longitudinales en fonction de la température dans le verre $B$ pour la fréquence de $11 \mathrm{MHz}$.

Les résultats obtenus pour les ondes longitudinales sont les suivants :

$$
\begin{array}{ll}
\text { Verre A } & E_{\mathrm{A}}=10 \pm 3 \mathrm{kcal} / \mathrm{mole} \\
\text { Verre B } & E_{\mathrm{A}}=14 \pm 3 \mathrm{kcal} / \mathrm{mole} .
\end{array}
$$

$\mathrm{Si}$ on compare ces énergies d'activation à celles déjà trouvées [5], on constate que la valeur obtenue pour le verre $\mathrm{A}$ est plus faible, et qu'il y a un bon accord pour le verre $B$.

Il faut toutefois remarquer que des calculs, dans un domaine de fréquence aussi étroit, sont peu précis ; une faible erreur sur la température du pic entraîne une grande variation de $E_{\mathrm{A}}$.

4.3 Épaulement. - Le verre A présente sur le côté basse température du pic de relaxation des ions sodium un épaulement. L'énergie d'activation apparente calculée par décomposition du pic est :

$$
E_{\mathrm{A}}=4 \pm 2 \mathrm{kcal} / \mathrm{mole} \text {. }
$$

A une énergie d'activation aussi faible, ne peut être assimilée une relaxation d'ion alcalin.

Kurkjian et Krause [11] trouvent un pic bien résolu à température ambiante dans le verre de $\mathrm{B}_{2} \mathrm{O}_{3}$ volontairement hydraté. Ils notent [10] que le même effet se produit dans les verres de borates de sodium. Avec l'augmentation de la teneur en alcalin, le pic s'estompe peu à peu pour laisser apparaître le pic des alcalins. Aucune tentative n'ayant été faite pour éliminer l'eau lors de la fusion, l'eau de structure semble être la cause de l'apparition de cet épaulement.

Strakna et Savage [12] trouvent une valeur de $6,3 \mathrm{kcal} / \mathrm{mole}$ pour le même phénomène.

4.4 Distribution Des temps de Relaxation. - A partir des relations $[2,5,6]$, les parties imaginaires et réelles des modules $M^{*}, G^{*}$ et $K^{*}$ ont été calculées pour la fréquence de $11 \mathrm{MHz}$ dans le verre $\mathrm{A}$ (Fig. 4).

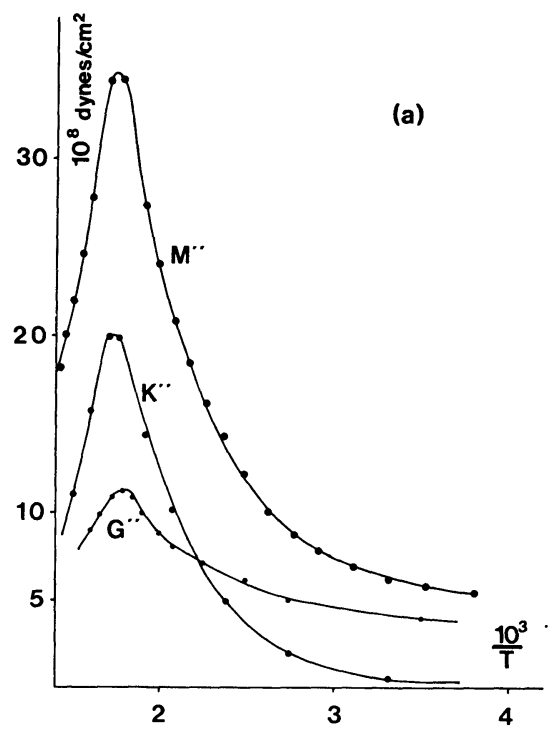

FIG. 4a. - Variation de la partie imaginaire des modules $M$, $G, K$, en fonction de l'inverse de la température.

Les parties réelles et imaginaires du module $M^{*}$ son montrées (Fig. 5) pour le verre B. En supposant u distribution log-normale des temps de relaxat: 


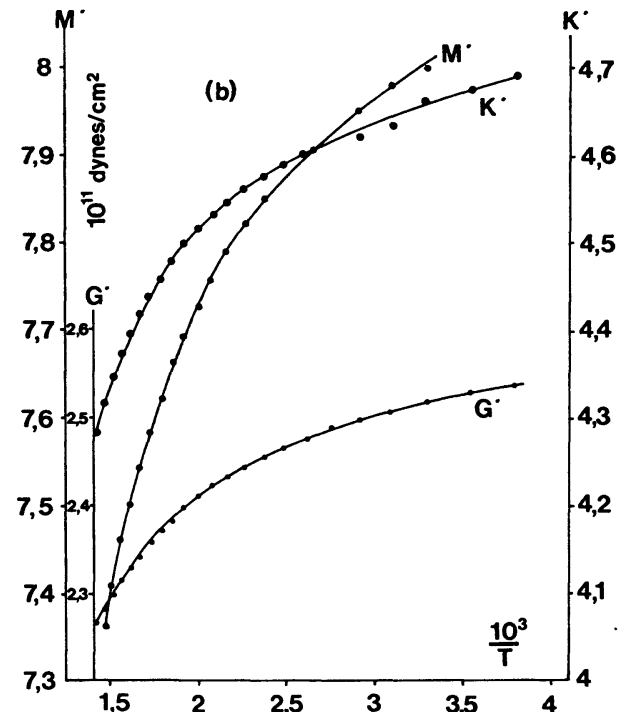

Fig. 4b. - Variation de la partie réelle des modules $M, G, K$ en fonction de l'inverse de la température.

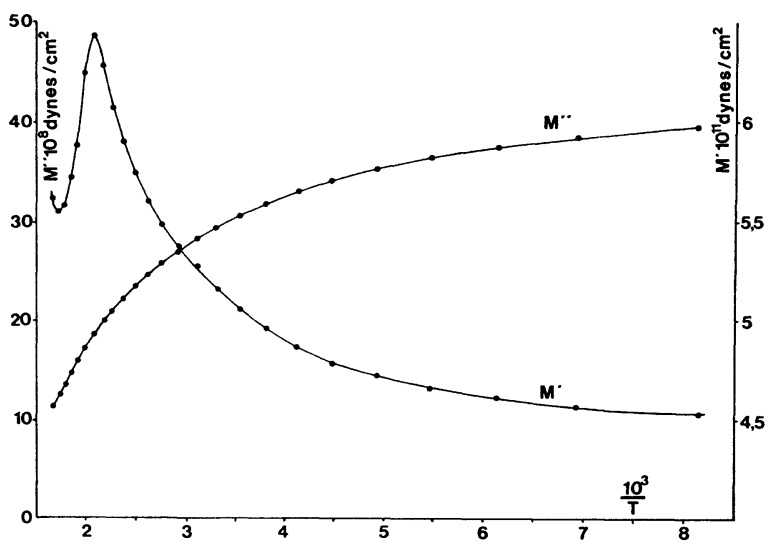

Fig. 5. - Variation des parties réelles et imaginaires du module $M$ pour le verre B en fonction de l'inverse de la température.

le paramètre $\beta$ peut être calculé pour chacun des modules à partir de la largeur à mi-hauteur des courbes $M^{\prime \prime}, G^{\prime \prime}$ et $K^{\prime \prime}$ en fonction de $1 / T$.

Les valeurs obtenues sont les suivantes :

$$
\begin{aligned}
0,62 & <\Delta(1 / T)_{M^{\prime \prime}}<0,635 \times 10^{-3} \text { degré }^{-1} \\
0,61<\Delta(1 / T)_{G^{\prime \prime}} & <0,65 \\
0,59 & <\Delta(1 / T)_{K^{\prime \prime}}<0,63
\end{aligned}
$$

où $\Delta(1 / T)$ représente la largeur à mi-hauteur de la partie imaginaire du module écrit en indice.

Aux erreurs expérimentales près, les valeurs trouvées sont donc identiques et la distribution des temps de relaxation à une fréquence donnée est caractérisée par une valeur unique de $\beta$. A partir des valeurs tabulées [6], on déduit que $\beta$ est alors égal à 2,15 .

Le même calcul réalisé pour le verre $\mathrm{B}$ donne :

$$
0,88<\Delta(1 / T)_{M^{\prime \prime}}<0,90 \text { et } \beta=3,4 .
$$

Puisque $\beta$ est indépendant du mode de propagation de l'onde, on peut analyser la variation de $\beta$ en fonction de la fréquence en utilisant des appareils faisant intervenir des modules quelconques. Cela a été fait (Fig. 6) sur les deux verres étudiés en complétant les résultats déjà obtenus [5].

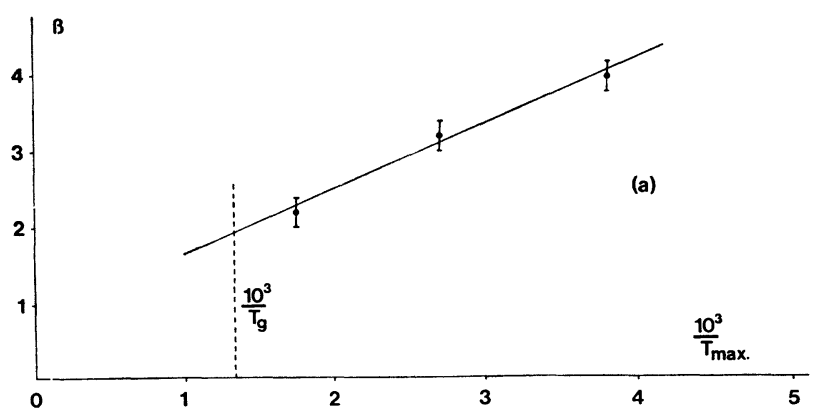

FIG. $6 a$. - Variation de $\beta$ en fonction de l'inverse de $T_{\max }$ pour le verre $\mathrm{A}$.

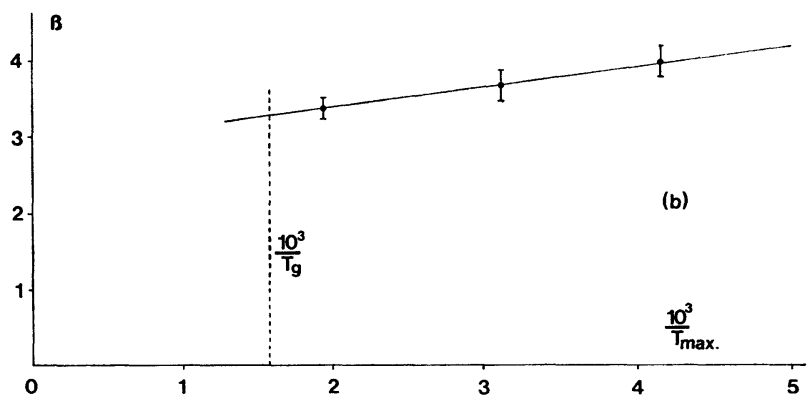

Fig. $6 b$. - Variation de $\beta$ en fonction de l'inverse de $T_{\max }$ pour le verre $B$.

La variation de $\beta$ avec la température $T_{\max }$ du maximum du pic de relaxation ne peut donc être attribuée qu'aux distributions respectives de l'enthalpie et de l'entropie d'activation.

5. Conclusion. - En assimilant la diffusion sous contrainte des ions alcalins au passage d'un ion d'un puits de potentiel à un autre, Mills [13] suggère que «la relaxation en torsion impliquerait à la fois la torsion et l'allongement des liaisons pour abaisser la barrière d'énergie entre les sites équivalents pour les ions sodium. Au contraire, la relaxation en volume n'impliquerait que des modes d'allongement. Puisque ces derniers modes sont dépendants de la séparation interionique qui tend à être plus précisément définie dans un verre que les angles de liaison, un spectre plus étroit de relaxation pour les effets en volume devrait en résulter ».

En caractérisant le spectre de relaxation par la grandeur $\beta$, ce paramètre devrait donc prendre deux valeurs respectives pour une onde de cisaillement et une onde de compression volumique, à la même fréquence. Or les manipulations réalisées sur les verres précédemment étudiés montrent que ce paramètre de distribution reste constant pour une fréquence donnée. 
D'autre part, si la largeur du spectre de relaxation n'était fonction que du mode de propagation des ondes, les valeurs de $\beta$ mesurées pour les spectres de relaxation en torsion (Méthode du pendule et méthode de l'écho d'impulsion (pulse-écho) en ondes transverses) devraient être identiques. Il n'en est pas ainsi puisque $\beta$ varie de 4 à 2,15 (Fig. 6a). L'hypothèse de Mills ne peut donc être retenue.

Le mouvement respectif des ions sodium n'est donc pas mieux défini dans le cas d'une onde de compression que dans le cas d'une onde de torsion. Vis-à-vis de la relaxation sous contrainte des ions alcalins, le mode de propagation de l'onde ne joue aucun rôle. La variation du spectre de relaxation ne peut être expliquée que par une distribution des termes enthalpiques et entropiques de l'énergie d'activation.

Remerciements. - Les auteurs remercient M. Delettre (Laboratoire des Verres du C. N. R. S.) pour le polissage des échantillons.

\section{Bibliographie}

[1] Forry, K. E., J. Amer. Ceram. Soc. 40 (1957) 90.

[2] Yager, W. A., J. Appl. Phys. 7 (1936) 434.

[3] Copley, G. J. and OAKLeY, D. R., Phys. Chem. Glasses 9 (1968) 141.

[4] Moore, D. W. and Day, D. E., Phys. Chem. Glasses 12 (1971) 75.

[5] Phalippou, J., Masson, S., Boyer, A. and Zarzycki, J., J. Non Cryst. Solids 14 (1974) 178.

[6] Nowick, A. S. and BerRy, B. S., IBM, J. Res. Dev. 5 (1961) $297,312$.

[7] Kono, R., J. Phys. Soc. Japan 16 (1961) 1580.
[8] Truell, R., Elbaum, C. and Chick, B. B., Ultrasonic Methods in Solid State Physics (Academic Press, New York) 1969.

[9] Papadakis, E. P., J. Acoust. Soc. Amer. 42 (1967) 1045.

[10] Krause, J. T. and Kurkjian, C. R., Am. Ceram. Soc. Bull. 43 (1969) 649.

[11] Kurkjian, C. R. and Krause, J. T., J. Amer. Ceram. Soc. 49 (1966) 171.

[12] Strakna, R. E. and Savage, H. T., J. Appl. Phys. 35 (1964) 1445.

[13] Mills, J. J., Phys. Chem. Glasses 14 (1973) 16 